\title{
Excessive growth hormone expression in bGH transgenic mice adversely alters bone architecture and quality
}

SV. Lim 1 , M. Marenzana ${ }^{2}$, EO. List ${ }^{3}$, JJ. Kopchick ${ }^{3}$, M. Korbonits ${ }^{4}$, C. Chenu ${ }^{1}$

'Veterinary Basic Sciences, Royal Veterinary College, London, UK, ${ }^{2}$ Imperial College London, London, UK,

${ }_{3}^{3}$ Edison Biotechnology Institute, Ohio University, Athens, USA, ${ }^{4}$ Endocrinology, Barts and the London Medical School, London, UK

\section{Introduction}

Growth hormone $(\mathrm{GH})$ is one of the important anabolic hormones involved in regulation of longitudinal bone growth ${ }^{1}$ by causing chondrocyte proliferation at the epiphyseal cartilage in the growth plate region. Acromegaly is a disease with abnormal skeletal tissue and organ growth ${ }^{2}$. It is caused by excessive $\mathrm{GH}$ expression after puberty ${ }^{2}$. Acromegaly patients have higher prevalence of vertebral fractures despite having normal bone mineral density (BMD), suggesting that BMD alone in not a sufficient indicator of fracture risk. The cause of this high incidence of fracture remains ill-defined.

\section{Aims}

1. To evaluate the skeletal changes in giant bovine $\mathrm{GH}$ overexpressing transgenic mice (bGH)

2. To establish the usefulness of bGH mice as a mouse model for the study of skeletal changes in acromegaly.

\section{Methods}

Micro-CT analysis of tibia of bGH and Wild Type (WT) mice:

5-month-old male bGH and WT mice ( $n=7 /$ group) were provided by Prof. John Kopchick (Ohio University, USA). Tibia were scanned with high

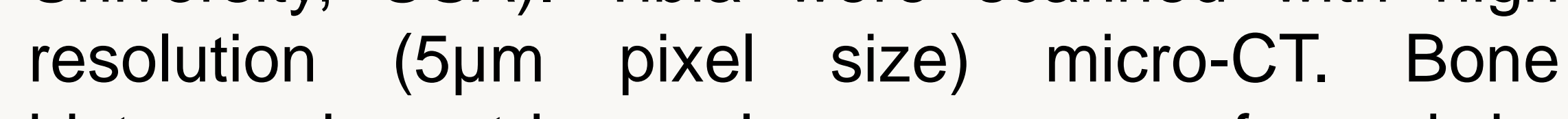
histomorphometric analyses were performed by SkyScan software (CT-Analyzer v.1.5.1.3). Trabecular bone parameters determined included bone volume (BV)/tissue volume (TV) percentage $(\mathrm{BV} / \mathrm{TV}), \%$ ), trabecular thickness (Tb. Th), trabecular bone pattern factor (TBPf). Cortical bone parameters determined included tissue perimeter (T. Pm), cross-sectional thickness (Cs. Th) and medullary area.

Measurement of BMD:

BMD analysis was performed in lumbar vertebrae (L4) using SkyScan software.

Mechanical testing:

Three-point bending tests on femora of WT and bGH mice were performed by as previously described $^{3}$. Calculations of bone mechanical properties including Young's modulus and ultimate stress.

Histological analysis of osteoclast activity:

Tibia from bGH and WT mice were fixed in $4 \%$ formaldehyde and decalcified. $8 \mu \mathrm{m}$-thick sections were stained for tartrate-resistant acid phosphatase (TRAP), a marker of osteoclast activity and counterstained with haematoxylin solution. TRAP staining was quantified using ImageJ software. Osteoclast activity was expressed as \% TRAP positive stain.

\section{Statistical analysis:}

Statistical analyses were performed using unpaired (two-tailed) $t$ test with GraphPad software, using $p<0.05$ as significant

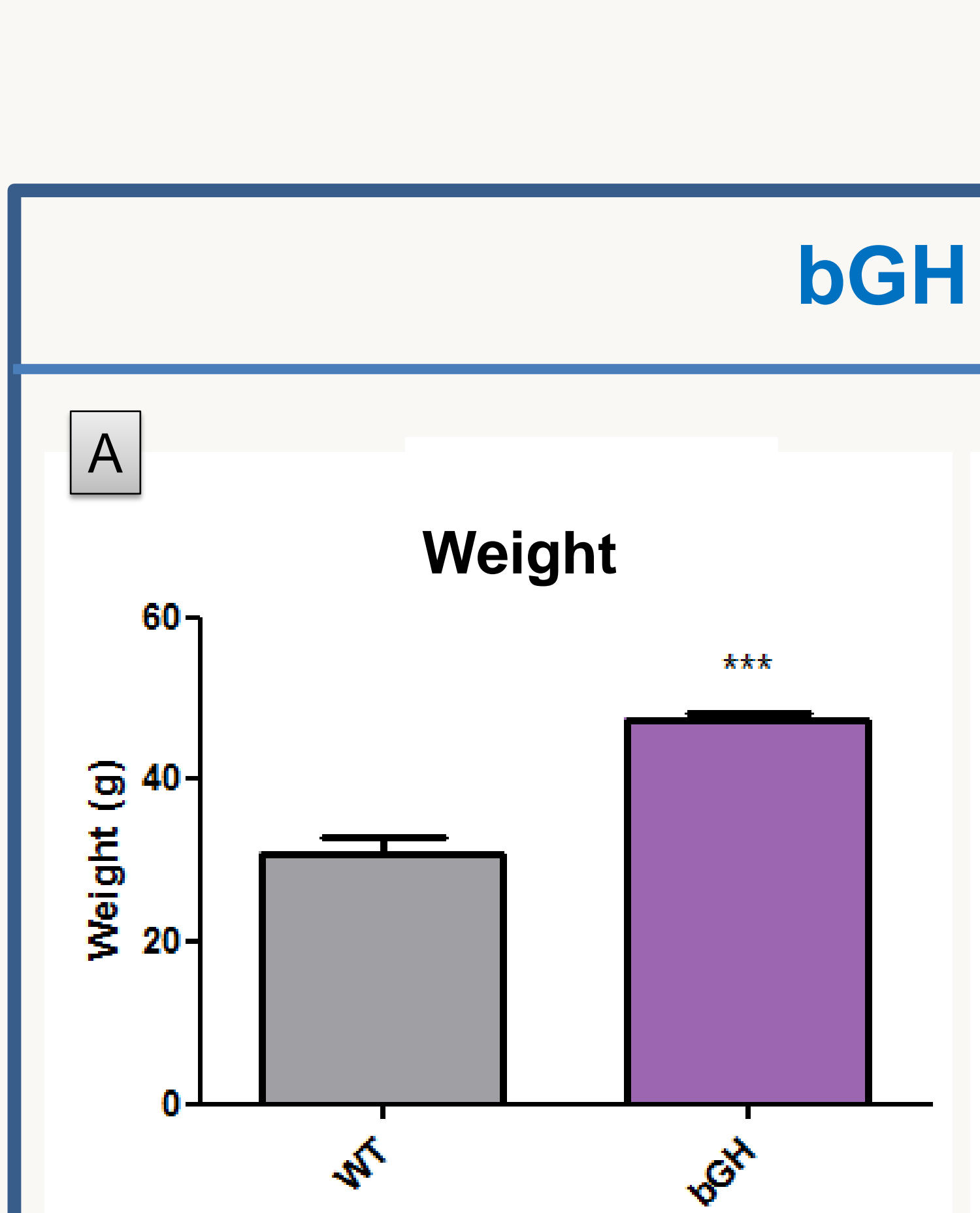

Fig 1. Weights of bGH and WT mice. Values are mean \pm SD of $n=7$ mice/group. ${ }^{* \star} p<0.00$

\section{Results}

GH mice have increased body weights and bone lengths

$B$
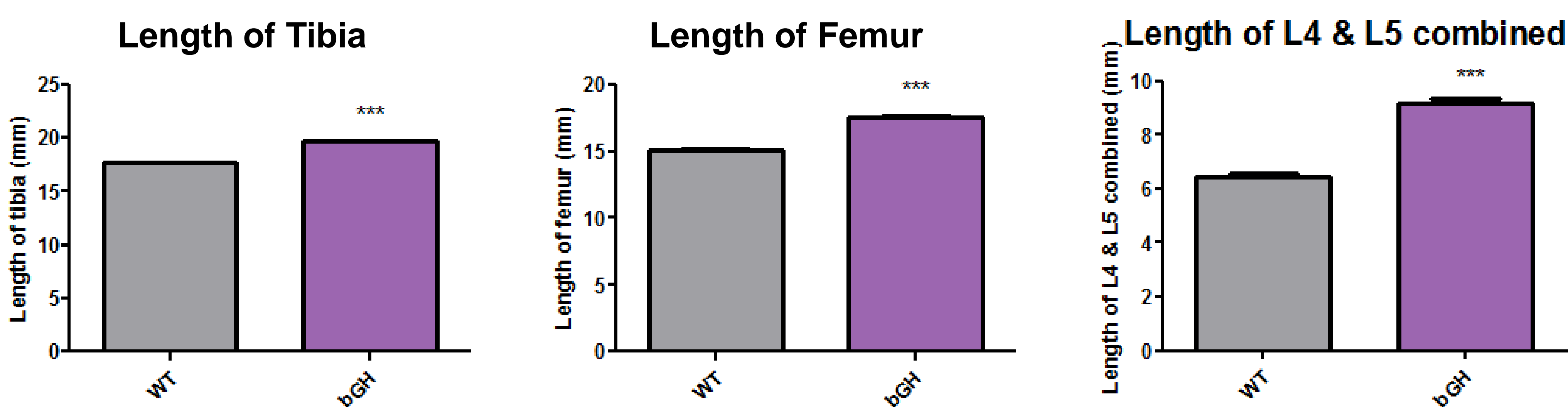

Fig 2. Lengths of bones (tibia, femur and vertebra) measured using micro-CT. Values are mean $\pm \mathrm{SD}$ of $n=7$ mice/group. ${ }^{* \star} p<0.001$

Bone architecture of bGH transgenic mice

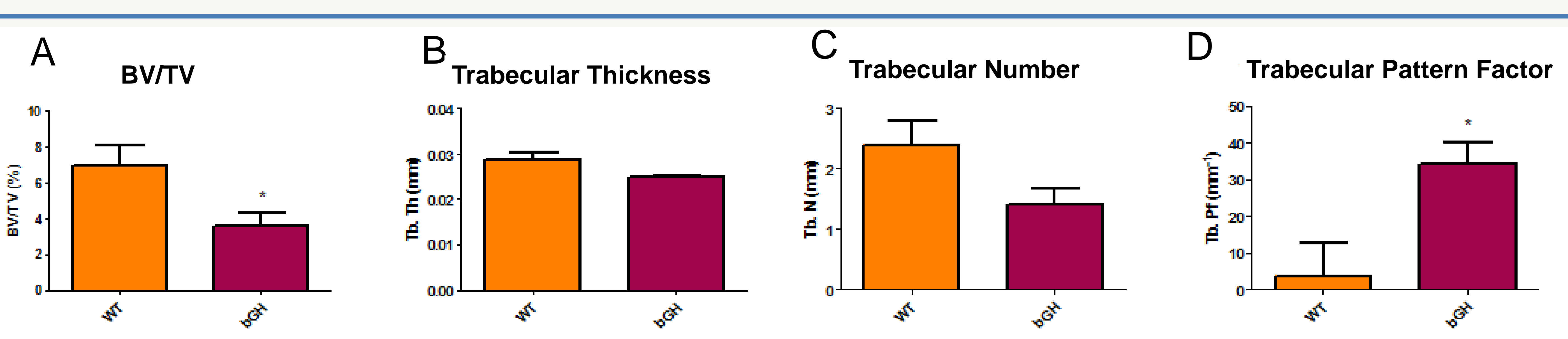

$E$

$\mathrm{F}$

G

Medullary Area
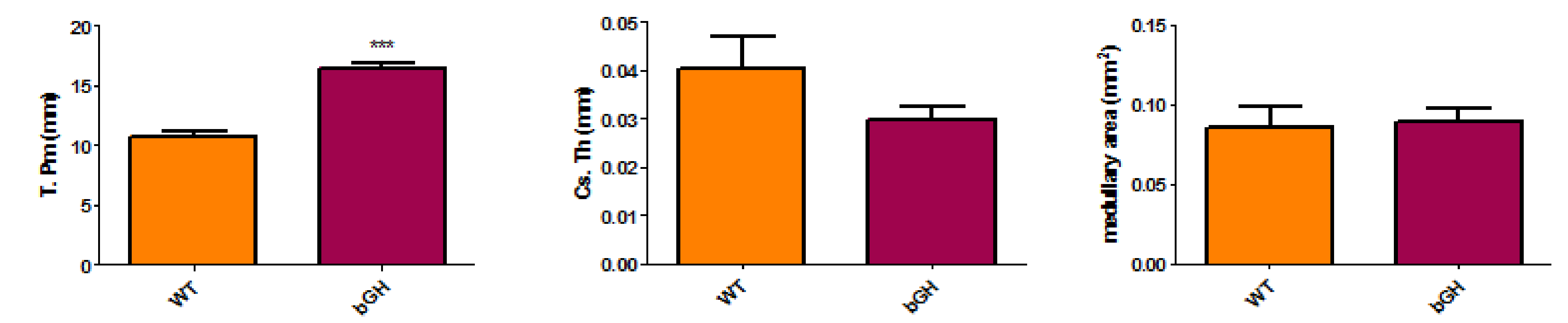

Fig 3. Trabecular and cortical microarchitecture in WT and bGH transgenic mice.

$(A, B, C, D) B V / T V(A), T b . T h(B), T b . N(C)$ and Tb. Pf (D) were measured in the proximal metaphysis of WT and bGH mice $(\mathrm{E}, \mathrm{F}, \mathrm{G}) \mathrm{T}$. Pm (E), Ct. Th $(\mathrm{F})$ and medullary area $(\mathrm{G})$ were measured in the mid-diaphysis cortical bone of WT and bGH mice Bars represent mean $\pm S D$ of $n=7$ mice/group. ${ }^{*} p<0.05,{ }^{* * *} p<0.001$

1. The decreases in BV/TV, trabecular number and cortical thickness in bGH mice compared to WT mice indicate that bGH mice have low bone mass.

2. The significant increase in trabecular pattern factor indicates less inter-trabecular connectivity, suggesting deterioration of trabecular bone in bGH mice.

\begin{tabular}{l}
\hline \multicolumn{2}{|c|}{ Bone mineral density } \\
\hline (n)
\end{tabular}

\section{Conclusion}

High serum GH levels negatively affect bone architecture and quality. bGH transgenic mice are a useful model to understand the mechanisms involved in the skeletal changes observed in acromegaly patients. 